\title{
225 In episodes of stridor
}

A Laryngismus stridulus is accompanied by a pyrexia.

B An inhaled foreign body should be excluded.

C A vocal cord paralysis may be present.

D If due to laryngomalacia the prognosis is good.

E In an infant with a normal appearance of the larynx an enlarged thymus may exist.

\section{Neural paralysis of the larynx}

A Aspiration is more likely if the superior laryngeal nerve is affected.

B The paralysed cord usually lies at a lower level.

C If due to bronchial carcinoma the primary pathology is easily diagnosed in most cases.

D Semon's law states that in a progressive lesion the adductors are affected before the abductors.

E The cartilage of Wrisberg becomes more prominent.

\section{Management of laryngeal paralysis}

A A unilateral recurrent laryngeal nerve paralysis may recover.

B If due to mediastinal spread from carcinoma of the oesophagus indicates a possibility of surgical cure.

C Laser anytenoidectomy is useful in unilateral lesions.

D Cordopexy obviates the need for a tracheostomy in bilateral recurrent nerve lesions.

E Teflon paste injection for unilateral paralysis is of little value.

\section{Voice disorders}

A Due to myotonia atrophica may produce phonaesthenia.

$B$ Of functional origin usually have an abnormal cough.

C Due to closure of the false cords are commonly psychogenic.

D Such as dysphonia plicae ventricularis may be caused by compensatory efforts in vocal disabilities.

E Due to ventricular band movements are best treated with speech therapy. 